\title{
Magnetic damping for maglev ${ }^{1,2}$
}

\author{
S. Zhu, Y. Cai, D.M. Rote* and S.S. Chen \\ Argonne National Laboratory, Argonne, \\ IL 60439, USA
}

Received 3 March 1998

Magnetic damping is one of the important parameters that control the response and stability of maglev systems. An experimental study to measure magnetic damping directly is presented. A plate attached to a permanent magnet levitated on a rotating drum was tested to investigate the effect of various parameters, such as conductivity, gap, excitation frequency, and oscillation amplitude, on magnetic damping. The experimental technique is capable of measuring all of the magnetic damping coefficients, some of which cannot be measured indirectly.

\section{Introduction}

One of the key elements in controlling the dynamic characteristics of maglev systems is magnetic damping. Two types of magnetic damping can be introduced: active and passive. In the absence of any enhanced damping, the magnetic damping inherent in a magnet-moving conductor system is generally referred to as intrinsic magnetic damping. Several aspects of magnetic damping have been studied: magnetic damping as a function of velocity (Moon [9], Iwamoto et al. [7], Yamada et al. [11]); damping constant as a function of frequency (Saitoh et al., 1992); passive damping (Iwamoto et al. [7]); and the effects

\footnotetext{
${ }^{1}$ The submitted manuscript has been created by the University of Chicago as Operator of Argonne National Laboratory ("Argonne") under Contract No. W-31-109-ENG-38 with the U.S. Department of Energy. The U.S. Government retains for itself, and others acting on its behalf, a paid-up, nonexclusive, irrevocable worldwide license in said article to reproduce, prepare derivative works, distribute copies to the public, and perform publicly and display publicly, by or on behalf of the Government.

${ }^{2}$ This work was performed under the sponsorship of the U.S. Army Corps of Engineers and the Federal Railroad Administration through interagency agreements with the U.S. Department of Energy.

*Corresponding author. E-mail: don_rote@qmgate.gov.
}

of enhanced passive and active damping on a test vehicle suspended and guided by the interaction of superconducting magnets interacting with a continuous sheet guideway (Coffey [4]). It is reasonable to say that damping characteristics under various conditions are still not well characterized.

The oscillation of a magnetic field source near a stationary conductor normally results in damping forces due to induced circulating currents in the conducting body (eddy currents). However, if the field source and conductor have a relative velocity transverse to the vibration, it is possible for the translational motion to add energy into the vibrational degree of freedom through negative magnetic damping (Moon [9]).

A magnetic lift force, $F_{\mathrm{L}}$, acting on a moving magnet over a thin-sheet conductor (thickness is $\Delta$, conductivity is $\sigma$ ) with a clearance of $h$ can be defined as

$$
F_{\mathrm{L}}=\frac{\mu_{0} I^{2}}{4 \pi h} \frac{v^{2}}{w^{2}+v^{2}},
$$

where $I$ is current filament, $\mu_{0}$ is the permeability of free space, $v$ is the velocity normal to the current filament and $w$ is a characteristic velocity given by $w=2 /\left(\mu_{0} \sigma \Delta\right)$. For a stationary conductor, $v=0$, it is well known that the damping forces will be produced due to the creation of eddy currents in the conductor. However, for a large enough velocity $v$, this dissipative force can become destabilizing. The subject of eddy current damping in the context of levitation problems was studied by Davis and Wilkie [5], Iwamoto et al. [7], Moon [8], and Baiko et al. [1]. When the levitation height varies sinusoidally, the perturbed lift force component, $F_{\mathrm{L}}^{\prime}$, proportional to sinusoidal height $u(t)$ with respect to the original height $h_{0}$ is found to be

$F_{\mathrm{L}}^{\prime}=\frac{\mu_{0} I^{2}}{4 \pi h_{0}}\left[\frac{v^{2}}{w^{2}+v^{2}} \frac{u}{h_{0}}+\frac{w^{2}-v^{2}}{\left(w^{2}+v^{2}\right)} \frac{w^{2} \mathrm{~d} u}{\mathrm{~d} t}\right]$,

where the first term in Eq. (2) represents a magnetic stiffness term and the second term, $\gamma$, represents a linear "viscous" damping proportional to the vertical velocity $\mathrm{d} u / \mathrm{d} t$ 


$$
\gamma=\frac{\mu_{0} I^{2} w}{4 \pi h_{0}} \frac{\left(w^{2}-v^{2}\right)}{\left(w^{2}+v^{2}\right)^{2}}
$$

When $v>w$, the damping can become negative from Eq. (3).

Experiments on negative eddy current damping were first done by Iwamoto et al. [7], and later by Moon [8] and summarized by Moon [9], which showed the same tendency of Eq. (3) that the magnetic damping becomes negative when the velocity of the thin aluminum conductor exceeds the characteristic velocity $w$.

Magnetic damping can be analyzed or measured either directly or indirectly. It appears that most of the past studies are based on the indirect methods. Recently, a direct method was applied to a maglev system by Chen et al. [3]. This direct method is capable of measuring self-induced and mutual magnetic damping. In a study of maglev response, all magnetic damping must be quantified; without damping values, it is difficult to predict the response of maglev systems.

This paper presents two series of tests designed to quantify passive magnetic damping. The purpose was to determine the effect of various parameters, such as conductivity, gap, excitation amplitude, and oscillation frequency, on magnetic damping. Once magnetic damping and stiffness are known, they can be applied to maglev systems.

\section{Experimental setup}

A schematic diagram of the test setup is shown in Fig. 1. It includes a rotating drum, shaker, force transducer, and displacement transducer. The drum is covered with an aluminum sheet, $26.99 \mathrm{~cm}$ in diameter, $14.61 \mathrm{~cm}$ wide, and $0.635 \mathrm{~cm}$ thick. The rotating speed can vary from 0 to $3500 \mathrm{rpm}$ with a velocity of 1 to $50 \mathrm{~m} / \mathrm{s}$. The shaker provides proper excitation force at given frequencies, and the impedance transducer measures the displacement of the supporting bar.

The force transducer used to measure the total force is shown in Fig. 2(a). The magnet, $2.54 \times 5.08 \times$ $0.318 \mathrm{~cm}$, is connected with copper brackets to an aluminum plate, $5.0 \times 7.6 \times 0.8 \mathrm{~cm}$, and then supported by an aluminum bar, $2.64 \mathrm{~cm}$ wide, $22.86 \mathrm{~cm}$ long, and $1.27 \mathrm{~cm}$ thick (Fig. 2(b)). The aluminum bar is attached to the shaker at one end and to the magnet and aluminum plate at the other. One set of strain gauges is placed on the smaller section of the aluminum bar to measure the force due to the excitation at other end. The general principle in the experimental setup to mea-

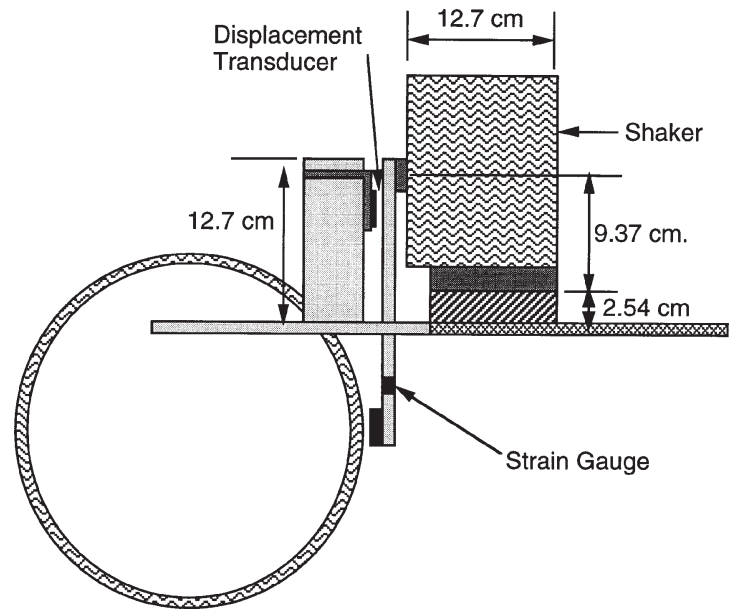

Fig. 1. Schematic diagram of test setup.

sure motion-dependent magnetic forces is the same as that for motion-dependent fluid forces (Chen et al. [2]).

Without magnetic field, the force transducer is calibrated by a dynamic method. The supporting bar is fairly rigid with a natural frequency of $>100 \mathrm{~Hz}$. For a given excitation frequency $f$ and amplitude $u_{0}$, imposed on the supporting bar by the shaker, we can calculate the inertia force of the magnet, aluminum plate, and support structure $F_{0}$. A typical result is given by $F_{0} / u_{0}=0.83837 f^{2.0098}$. Theoretically, the inertia force should be proportional to the square of the excitation frequency. The measured power of $f, 2.0098$, is very close to 2 . The calibration constant of the force transducer can be calculated from the inertia force, displacement, and strain gauge reading. The force transducer measures the total force with a sensitivity of $\approx 1 \mathrm{v}$ for $120 \mathrm{~g}$ of force acting on the middle of the magnet and damping plate.

The error resulting from this method of calibration is very small. The frequency range of interest is below $6 \mathrm{~Hz}$ while the natural frequency of the supporting bar is above $100 \mathrm{~Hz}$. The error due to the phase difference between the displacement and measured force (including inertia force and damping force) is practically zero because of very small frequency ratios, smaller than 0.05 (Harris and Crede [6]). The error of the power of frequency $f$, to which $F_{0} / u_{0}$ is proportional, is less than $0.5 \%$.

\section{Test cases and data analysis}

The detailed arrangement of the magnet and aluminum plate is shown in Fig. 2(b). Two cases were tested. 


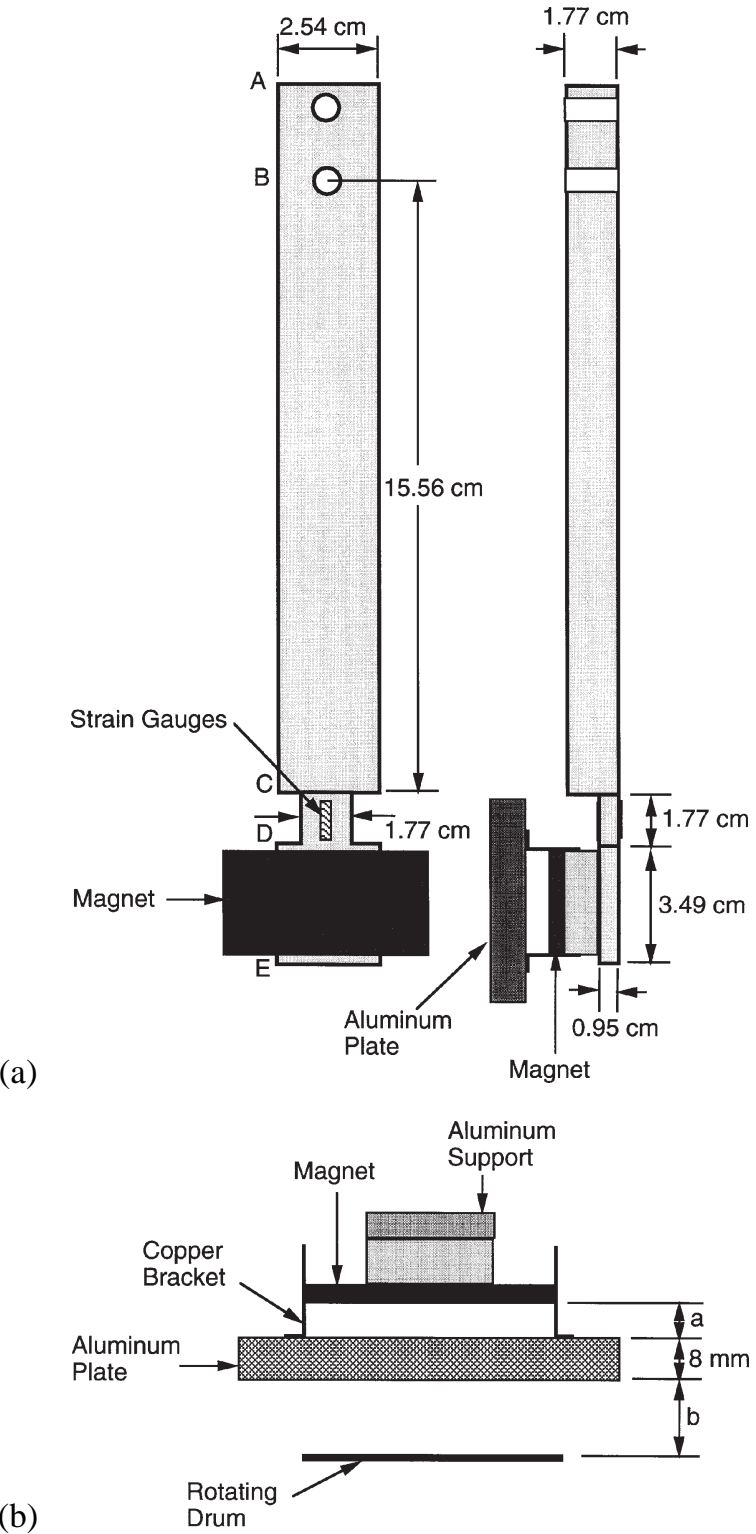

Fig. 2. Force transducer and magnet support: (a) overview, (b) active element.

Case A: Magnet and pure aluminum plate.

A $5.0 \times 7.6 \times 0.8 \mathrm{~cm}$ aluminum plate, with a purity of $99.999 \%$, was attached to the magnet. The gaps between aluminum plate and magnet, $a$, and aluminum plate and rotating drum, $b$, were set at various values. Five tests were performed:
A1
$a=1 \mathrm{~mm}, \quad b=3 \mathrm{~mm}$
A2 $a=1 \mathrm{~mm}, \quad b=5.5 \mathrm{~mm}$;
A3 $a=6 \mathrm{~mm}, \quad b=3 \mathrm{~mm}$;

$$
\begin{array}{ll}
\text { A4 } & a=3.5 \mathrm{~mm}, \quad b=5.5 \mathrm{~mm} ; \\
\text { A5 } & a=1 \mathrm{~mm}, \quad b=8 \mathrm{~mm} .
\end{array}
$$

Case B: Magnet and ordinary aluminum plate.

An aluminum plate $(6061-\mathrm{T} 6), 5.0 \times 7.6 \times 0.8 \mathrm{~cm}$, was attached to the magnet. Three tests were performed.

$$
\begin{array}{ll}
\mathrm{B} 1 & a=1 \mathrm{~mm}, \quad b=3 \mathrm{~mm} ; \\
\mathrm{B} 2 & a=1 \mathrm{~mm}, \quad b=5.5 \mathrm{~mm} ; \\
\mathrm{B} 3 & a=6 \mathrm{~mm}, \quad b=3 \mathrm{~mm} .
\end{array}
$$

In each test, the shaker provided an excitation at a given frequency with a specific amplitude. The displacement of the aluminum support, measured by the displacement transducer, and the forces consisting of the inertia force of the active element (magnet, aluminum plate, copper bracket, and aluminum support below the strain gauges) and magnetic force, measured by the strain gauges, were measured simultaneously. The RMS magnitude of displacement and force, as well as the phase angle between them at the excitation frequency, were obtained. Magnetic stiffness and damping can be calculated from these data. Each test was performed at several excitation frequencies for the whole range of rotating velocities.

The measured dynamic force is given as follows:

$$
F=-m \frac{\mathrm{d}^{2} u}{\mathrm{~d} t^{2}}-C \frac{\mathrm{d} u}{\mathrm{~d} t}-K u,
$$

where $u$ is the displacement, $m$ is the mass of the active element, and $C$ and $K$ are, respectively, the damping coefficient and stiffness to be determined. Note that $m$ should also include magnetic mass. However, because the excitation frequency of maglev is fairly low, the magnetic mass will be very small and is negligible (Iwamoto et al. [7]).

If we let the RMS values of the displacement and force be $d_{0}$ and $f_{0}$, respectively, and the phase angle between the two be $\phi, C$ and $K$ are given by

$$
\begin{aligned}
& C=f_{0} \sin (\phi) /\left(\omega d_{0}\right), \\
& K=m \omega^{2}-\frac{f_{0}}{d_{0}} \cos (\phi),
\end{aligned}
$$

where $\omega$ is the circular frequency of oscillations. Magnetic damping and stiffness are calculated from Eqs (5).

\section{Experimental results}

Typical results are presented in Figs 3 and 4 for test A5, with the excitation frequency equal to $6 \mathrm{~Hz}$. 

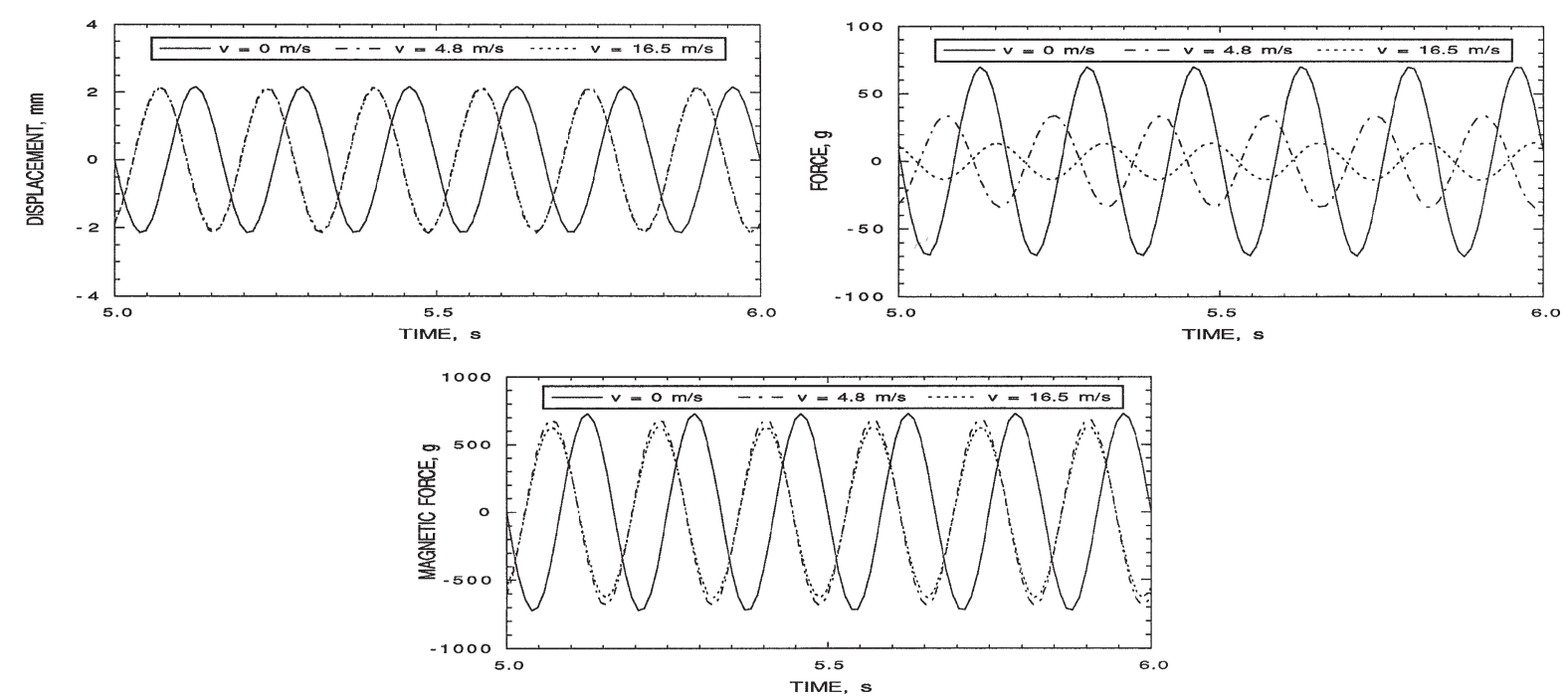

Fig. 3. Displacement, total force, and magnetic force as a function of time at an excitation of $6 \mathrm{~Hz}$, test A5.

In Fig. 3, the time histories of displacement, measured force, and magnetic force at three different velocities, $0,4.8$, and $16.5 \mathrm{~m} / \mathrm{s}$, are given. The measured force includes inertia and magnetic force (i.e., measured force $=$ inertia force + magnetic force). The magnetic force was determined by subtracting the inertia force from the measured force. The inertia force was out of phase with the displacement, while the magnetic force was approximately in phase with displacement. In this case, inertia force and magnetic force were of approximately the same magnitude (Chen et al. [3]). Therefore, the measured force became fairly small. The measured force was approximately in phase with the displacement when the speed was 0 and $4.8 \mathrm{~m} / \mathrm{s}$, whereas it was out of phase with the displacement when the speed was $16.5 \mathrm{~m} / \mathrm{s}$. The transition occurred near the characteristic speed of the aluminum sheet on the rotating drum.

Figure 4 shows the RMS displacement of the support bar, RMS force measured by the strain gauge, and the phase angle between the two as a function of velocity. These data were obtained from the time histories of the displacement of the support bar and the measured force shown in Fig. 3. Magnetic damping and stiffness were calculated from Eqs (5). The RMS displacement decreased slightly with increasing velocity for a fixed input to the shaker. The measured RMS force changed significantly with velocity. Magnetic stiffness increased, while magnetic damping decreased with velocity. In this case, the magnetic damping was positive.

Tests at 2, 4, and $6 \mathrm{~Hz}$ were conducted for the whole range of rotating velocities for all test cases. The iner- tia force, which is proportional to the square of the excitation frequency, was reduced at 2 and $4 \mathrm{~Hz}$; the measured force was mainly attributed to magnetic force. For different excitation frequencies, the drastic change of phase angle between displacement and measured force also changed with excitation frequencies.

Figures 5 and 6 show the magnetic damping and stiffness for various tests as a function of velocity at three excitation frequencies. The following general characteristics were noted:

- Magnetic damping decreased with velocity at low velocity. At zero velocity, it was always positive. Once the velocity exceeded the characteristic velocity, its value showed a tendency to increase slightly with the velocity. The experimental results give the same curve tendency of Eq. (3) as plotted in Ref. of Moon [8] due to eddy current damping effects.

- Magnetic stiffness increased with velocity. At high velocities, its values were almost independent of velocity.

- Magnetic stiffness was independent of excitation frequency, whereas magnetic damping depended on excitation frequency, with its value increasing slightly with excitation frequency.

By comparing magnetic damping and stiffness of various cases, we can understand the effects of various parameters which may affect the eddy current damping (also see Eq. (3)). 

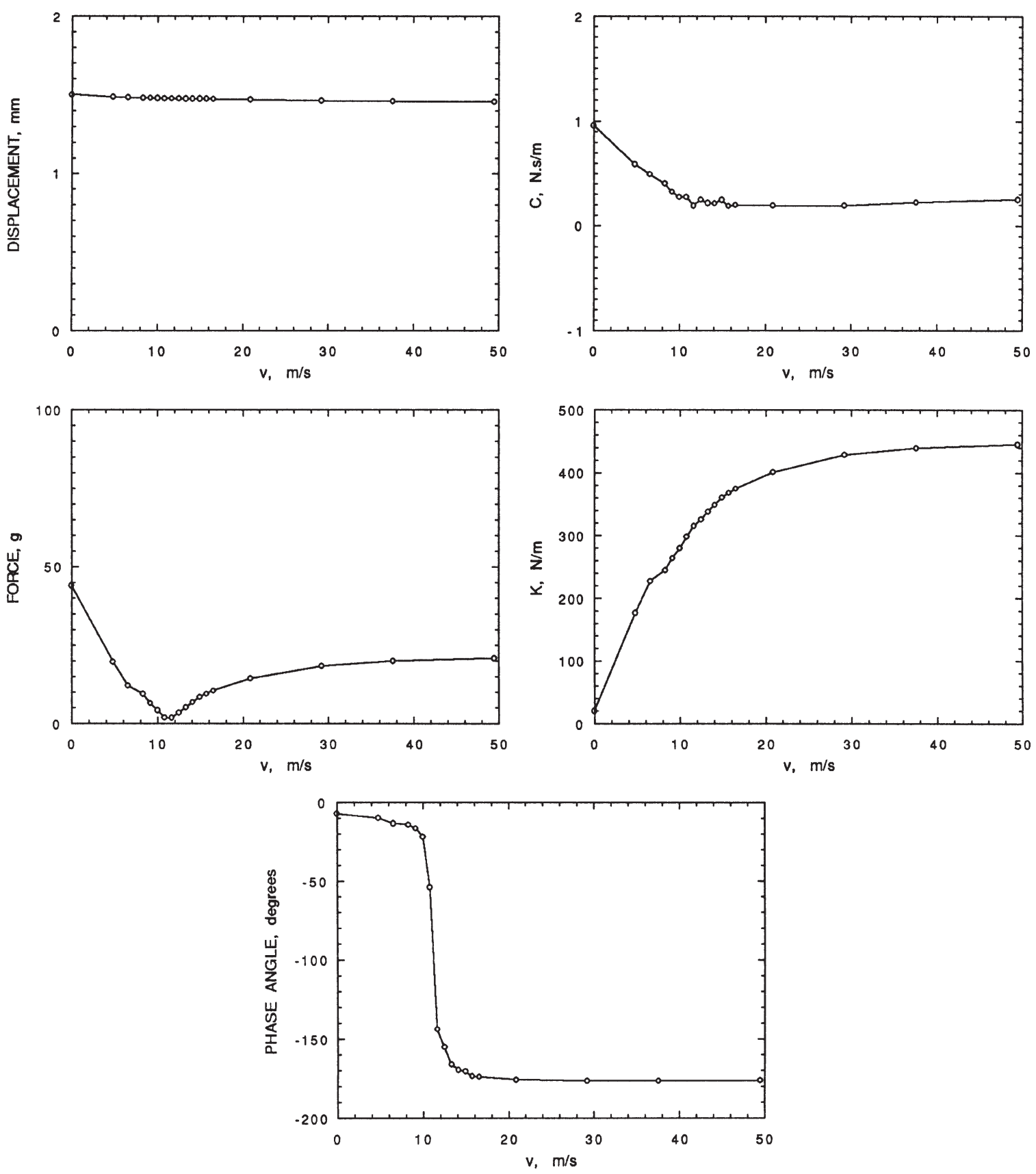

Fig. 4. RMS displacement and force, phase angle, and magnetic damping and stiffness excited at $6 \mathrm{~Hz}$, test A5.

\subsection{Conductivity}

Tests A1-A3 correspond to tests B1-B3; the difference is the aluminum plate. In series $\mathrm{A}$, the $\mathrm{pu}$ rity of the plate was $99.999 \%$ with the conductivity $=$ $61 \%$ IACS; in series B, it was 6061-T6 with the conductivity $=55 \%$ IACS. From Figs 5 and 6, the following characteristics were noted:
- Magnetic stiffness was about the same for various excitation frequencies in both test series. The purity of the aluminum plate did not affect magnetic stiffness.

- Magnetic damping for tests A2 and B2 were about the same. In these two tests, the aluminum plate was $5.5 \mathrm{~mm}$ from the drum. However, when the 

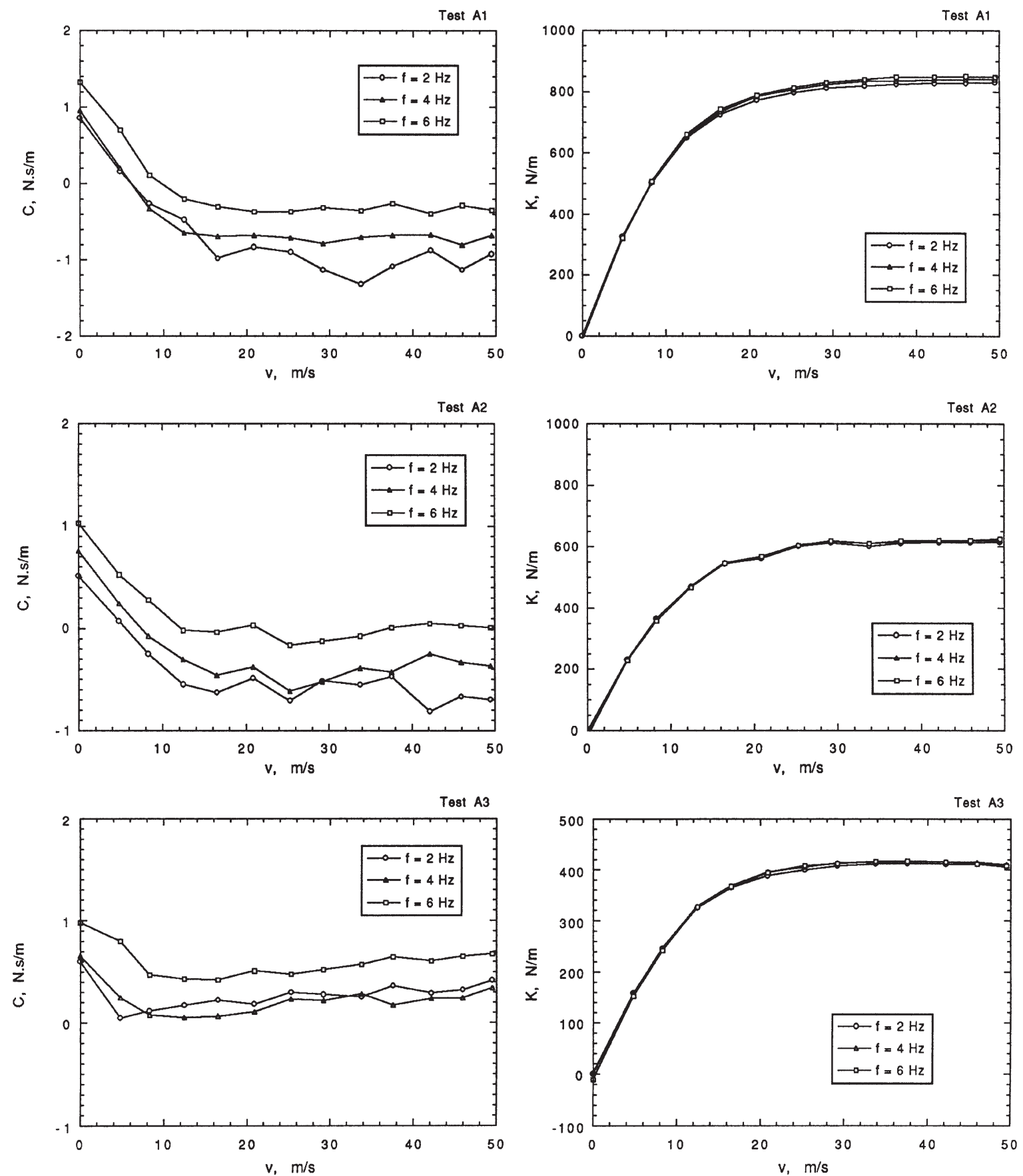

Fig. 5. Magnetic damping and stiffness for series A tests (continued on the next page).

gap was $3 \mathrm{~mm}$, in tests $\mathrm{A} 1, \mathrm{~A} 3, \mathrm{~B} 1$, and B3, magnetic damping values of the series $A$ tests were larger than those of the series B tests; hence the aluminum plate with higher purity provided greater damping.

\subsection{Gap}

- In tests A3-A5, the gap between the magnet and drum was kept constant, while the gap between the aluminum and the drum was varied from 3 

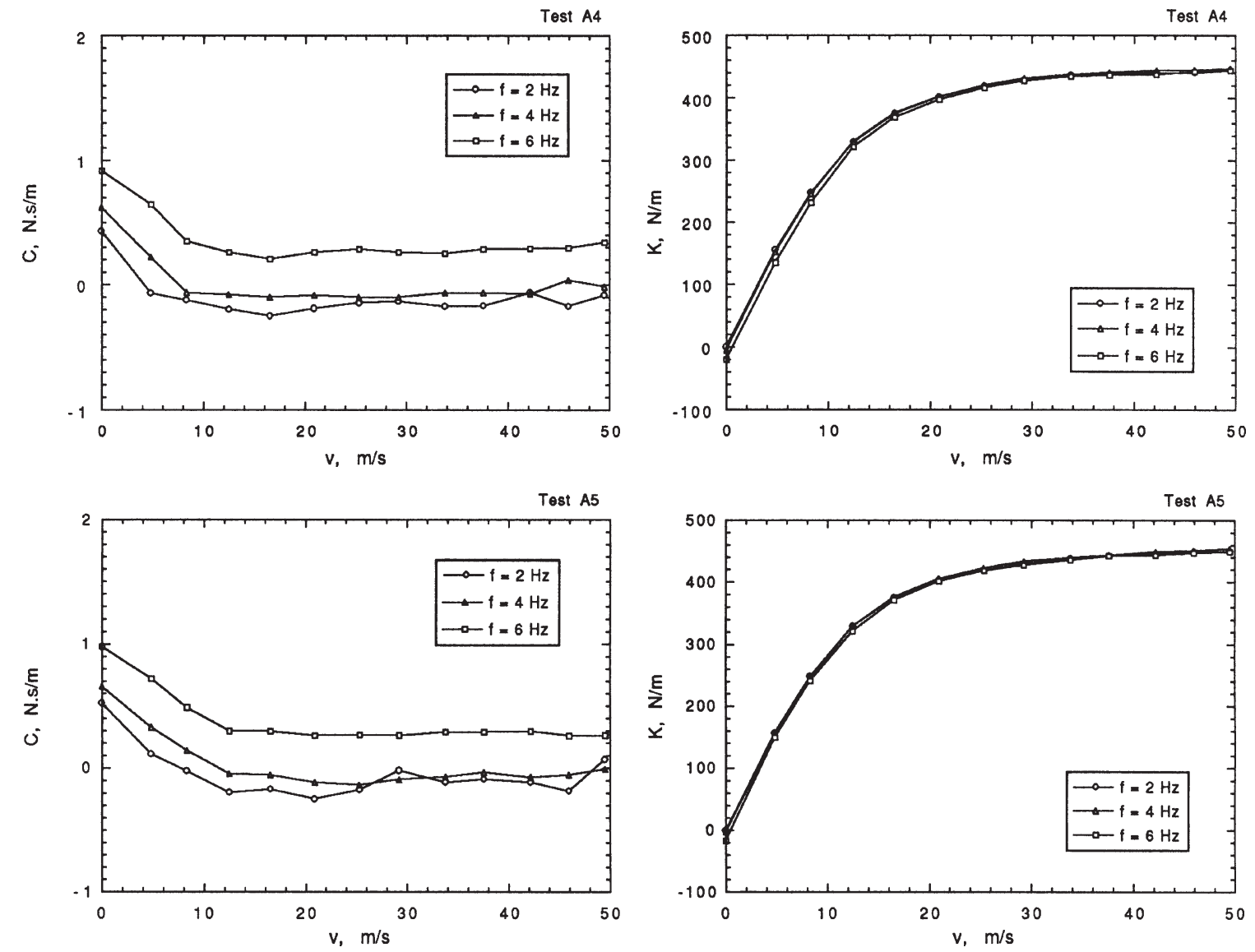

Fig. 5. (Continued.)

to 5.5 to $8 \mathrm{~mm}$. Magnetic stiffnesses for the various gaps were approximately the same. However, magnetic damping values depended on the gap. As the aluminum plate was moved closer to the drum, magnetic damping increased. The magnetic damping values for the 3- and 5.5-mm gaps were larger than those for the 8-mm gap.

- In tests A1 and A3, or tests A2 and A4, the gap between the aluminum and drum was fixed while the gap between the magnet and the aluminum plate was varied. We can also compare the magnetic damping values for the two sets of tests. When the magnet was further away from the drum, magnetic stiffness decreased and magnetic damping increased.

\subsection{Frequency}

The effect of excitation frequency on magnetic damping and stiffness is presented in Fig. 7 for 4.8 m/s.
Other velocities were also tested. Magnetic stiffness was practically independent of frequency, whereas magnetic damping increased slightly with frequency.

\subsection{Excitation amplitude}

The magnetic stiffness and damping for test B3 as a function of excitation amplitude at several velocities were measured. Regardless of the velocity, excitation amplitude affects neither magnetic stiffness nor damping in the parameter range tested. This means that a linear theory can be used if the displacement is small.

\section{Applications to maglev}

Once maglev damping and stiffness are known, they can be applied to vehicle dynamics. For example, consider a maglev vehicle, with mass $m$, moving on a guideway. The equation of motion is 

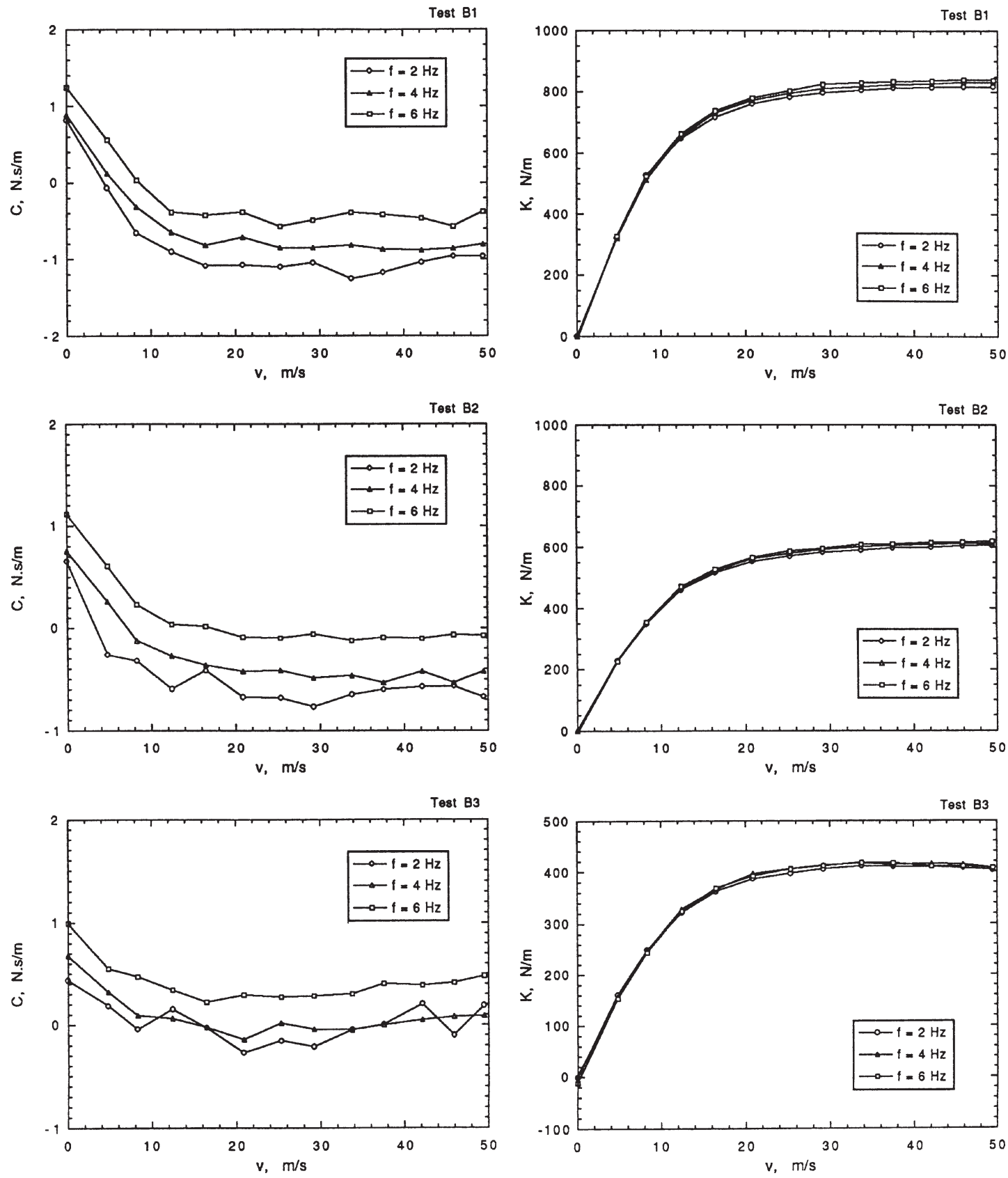

Fig. 6. Magnetic damping and stiffness for series B tests.

$$
m \frac{\mathrm{d}^{2} u}{\mathrm{~d} t^{2}}+\left(C_{\mathrm{s}}+C\right) \frac{\mathrm{d} u}{\mathrm{~d} t}+K u=q(t)
$$

where $m$ is the total mass of the vehicle; $C_{\mathrm{s}}$ is structural damping; $C$ is magnetic damping, including aerodynamic damping; $K$ is magnetic stiffness; and $q(t)$ is external excitation.
The natural oscillation frequency and modal damping ratio are, respectively,

$$
f=\frac{1}{2 \pi} \sqrt{\frac{K}{m}}, \quad \zeta=\frac{C_{\mathrm{s}}+C}{2 \sqrt{K m}} .
$$

For a given vehicle, $C$ and $K$ depend on clearance and velocity. With the data obtained in this experiment, we 

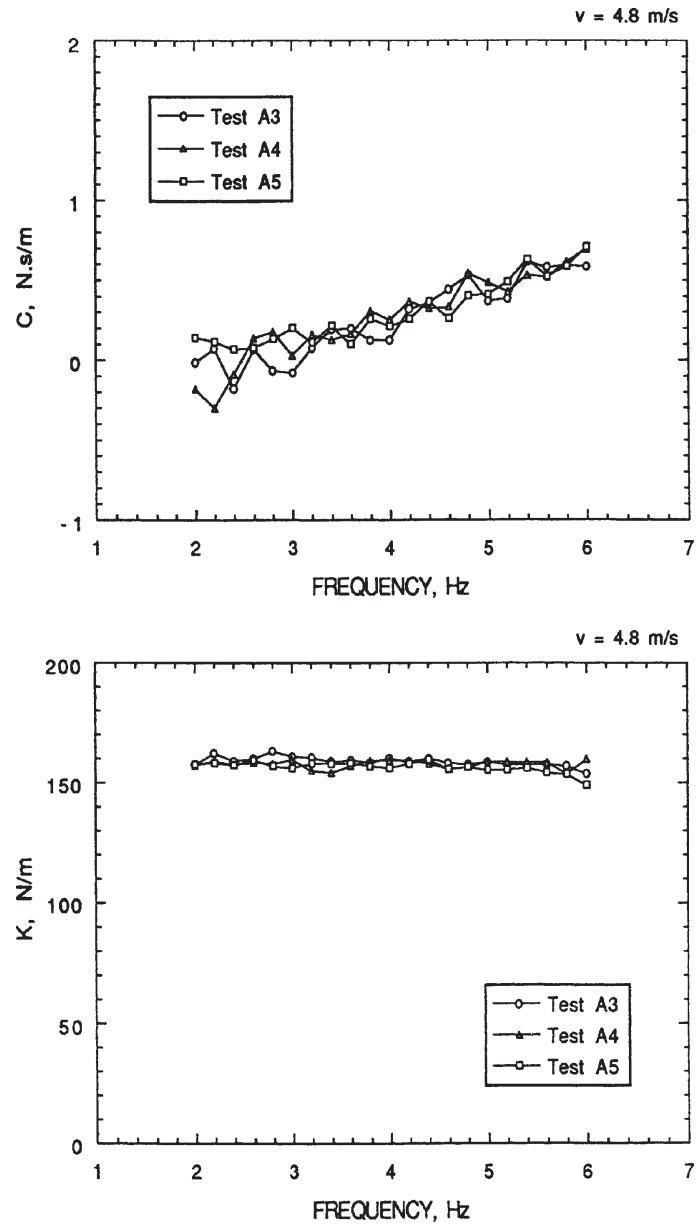

Fig. 7. Magnetic damping and stiffness as a function of excitation frequency for tests $\mathrm{A} 3-\mathrm{A} 5$, at $4.8 \mathrm{~m} / \mathrm{s}$.

can analyze the system characteristics. For example, magnetic damping and stiffness obtained in tests A1, $\mathrm{A} 2$, and A5 can be used to simulate a vehicle with a passive damping plate levitated on a guideway. Magnetic damping and stiffness depend on clearance $b$ (see Fig. 2(b)) and oscillation frequency $f$. From Fig. 5, we can obtain $C$ and $K$ at $37.6 \mathrm{~m} / \mathrm{s}$ as follows:

$$
\begin{aligned}
& b=1 \mathrm{~mm}, \\
& K=814.89+4.187 f+0.219 f^{2}, \\
& C=-1.494+0.199 f+0.001 f^{2}, \\
& b=5.5 \mathrm{~mm}, \\
& K=603.82+3.293 f-0.118 f^{2}, \\
& C=-0.109-0.282 f+0.05 f^{2}, \\
& b=8 \mathrm{~mm}, \\
& K=439.54+2.03 f-0.257 f^{2}, \\
& C=0.129-0.177 f+0.034 f^{2} .
\end{aligned}
$$

From Eqs (7) and (8), and from the static magnetic force, we can calculate the natural frequency and modal damping ratio as a function of $b$. Furthermore, the response of a maglev system can be predicted from Eq. (6) for a given excitation $q(t)$.

\section{Closing remarks}

A direct method was used to measure magnetic damping and stiffness during two series of tests. The effects of conductivity, gap, excitation amplitude, and excitation frequency which are related to the eddy current damping were investigated.

Once magnetic damping and stiffness are known, the dynamic response of maglev systems can be predicted. In addition, a passive damping plate can be introduced to improve ride quality and control stability. Other control techniques that are based on the characteristics of magnetic forces can also be applied to maglev.

The described technique will be very useful for measuring magnetic damping and stiffness coefficients of prototypes and will provide the necessary elements for predicting maglev response and controlling maglev systems.

\section{Acknowledgements}

This work was performed under the sponsorship of the U.S. Army Corps of Engineers and the Federal Railroad Administration through interagency agreements with the U.S. Department of Energy.

\section{References}

[1] A.V. Baiko, K.E. Voevodskii and V.M. Kochetkov, Vertical unstability of electrodynamic suspension of high speed ground transportation, Cryogenics 20 (1980), 271-276.

[2] S.S. Chen, S. Zhu and J.A. Jendrzejczyk, Fluid damping and fluid stiffness of a tube row in crossflow, J. Pressure Vessel Technol. 116 (1994), 370-383.

[3] S.S. Chen, S. Zhu and Y. Cai, On unsteady-motion theory of magnetic forces for maglev system, J. Sound Vibration 188(4) (1995), 529-543.

[4] H.T. Coffey, SRI magnetic suspension studies for high-speed vehicles, Adv. Cryogenic Eng. 19 (1974), 137-153.

[5] L.C. Davis and D.F. Wilkie, Analysis of motion of magnetic levitation systems: Implications, J. Appl. Phys. 42(12) (1971), 4779-4793.

[6] C.M. Harris and C.E. Crede, Shock and Vibration Handbook, McGraw-Hill, New York, 1976. 
[7] M. Iwamoto, T. Yamada and E. Ohno, Magnetic damping force in electrically suspended trains, IEEE Trans. Magn. 10(3) (1974), 458-461.

[8] F.C. Moon, Vibration problems in magnetic levitation and propulsion, in: Transportation without Wheels, E.R. Laithwaite and Paul Elek, eds, Scientific Books, London, 1977, pp. 123161.
[9] F.C. Moon, Superconducting Levitation, Wiley, New York, 1994.

[10] M. Nagai and S. Tanaka, Study on the dynamic stability of repulsive magnetic levitation systems, Soc. Mech. Eng. Int. J., Series III 35(1) (1992), 102-108.

[11] T. Yamada, M. Iwamoto and T. Ito, Magnetic damping force in inductive magnetic levitation system for high-speed trains, Elect. Eng. Japan 94(1) (1974), 49-54. 

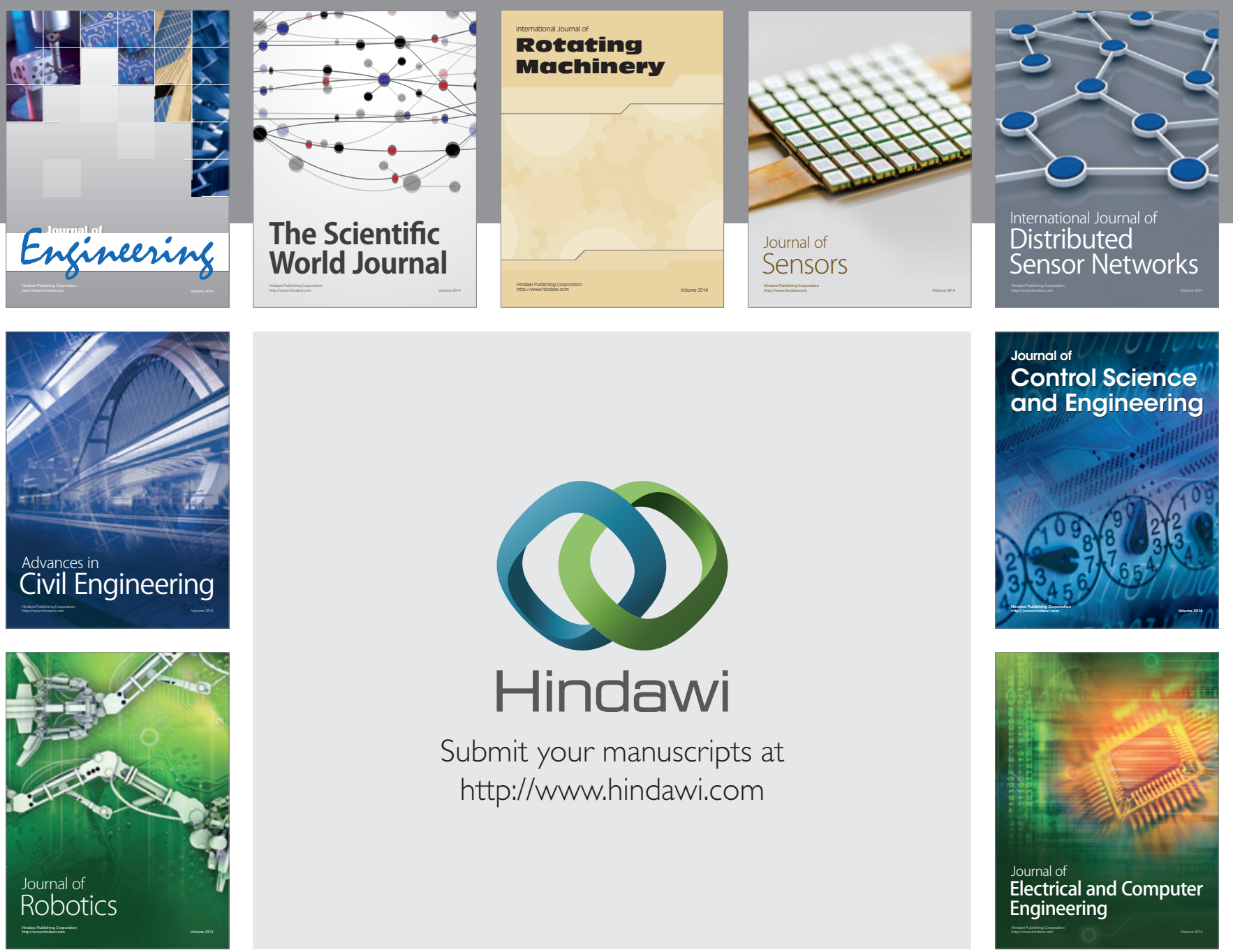

Submit your manuscripts at

http://www.hindawi.com
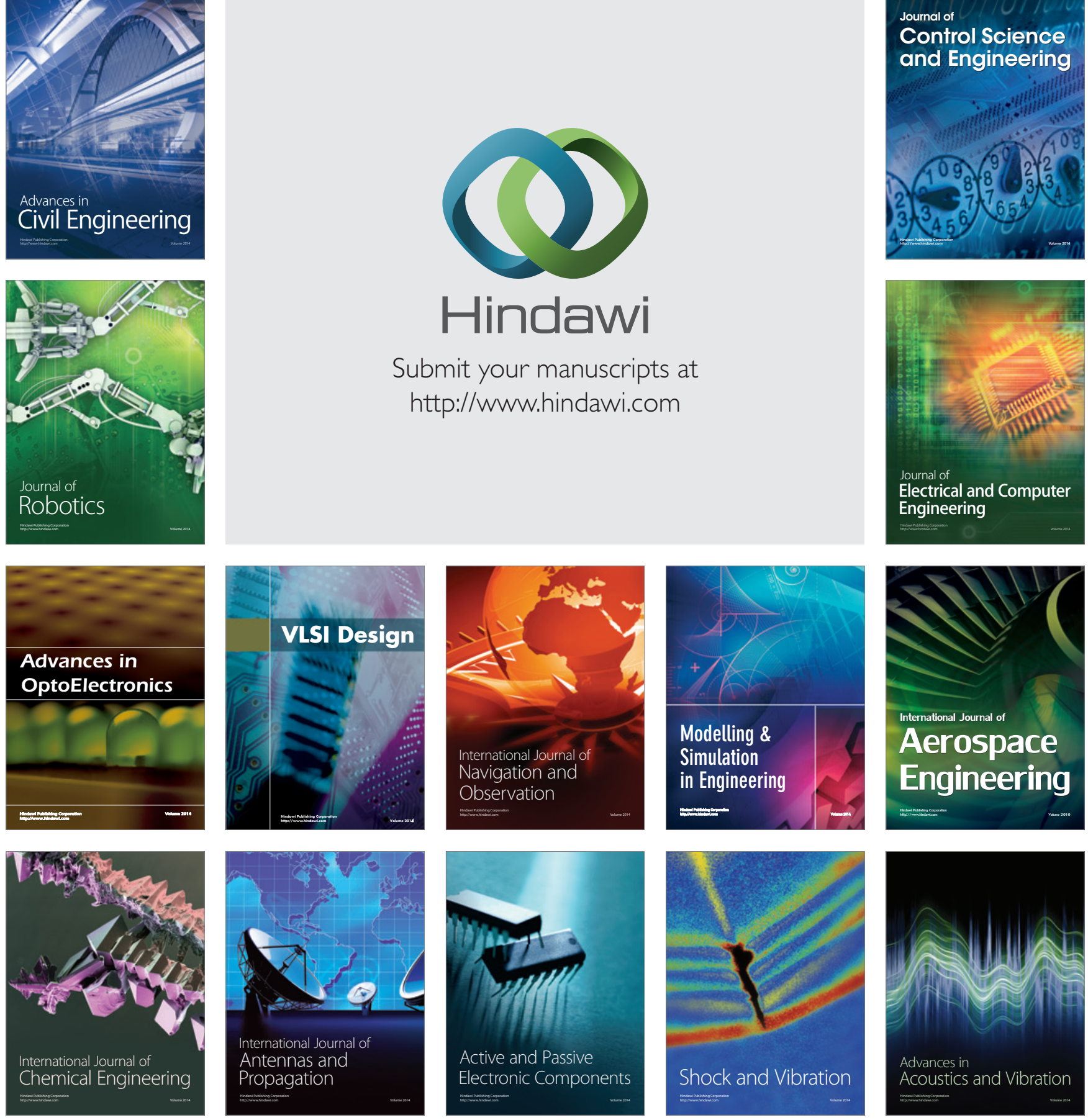